\title{
On some generalizations of dynamic Opial-type inequalities on time scales
}

\author{
Fatma M. KH ${ }^{1}$, A.A. El-Deeb2 ${ }^{2 *}$ A. Abdeldaim ${ }^{3,4}$ and Zareen A. Khan ${ }^{5}$
}

\section{${ }^{*}$ Correspondence:}

ahmedeldeeb@azhar.edu.eg

${ }^{2}$ Department of Mathematics,

Faculty of Science, Al-Azhar

University, Cairo, Egypt

Full list of author information is

available at the end of the article

\begin{abstract}
In this paper, we prove some new generalizations of dynamic Opial-type inequalities on time scales. From these inequalities, as special cases, we formulate some integral and discrete inequalities proved in the literature and also extend some obtained dynamic inequalities on time scales. The main results are proved by using some algebraic inequalities, Hölder's inequality, and a simple consequence of Keller's chain rule on time scales.
\end{abstract}

\section{Introduction}

In 1960, Opial [24] proved the following inequality:

$$
\int_{a}^{b}|x(t)|\left|x^{\prime}(t)\right| d t \leq \frac{b-a}{4} \int_{a}^{b}\left|x^{\prime}(t)\right|^{2} d t
$$

where $x$ is absolutely continuous on $[a, b]$ and $x(a)=x(b)=0$, and the constant $\frac{b-a}{4}$ is the best possible.

Equality holds in (1.1) if and only if

$$
x(t)=c(t-a), \quad \text { for } a \leq x \leq \frac{b-a}{2},
$$

and

$$
x(t)=c(b-t), \quad \text { for } \frac{b-a}{2} \leq x \leq b,
$$

where $c$ is a constant.

Opial's inequality together with its numerous generalizations, extensions, and discretizations has been playing a fundamental role in the study of the existence and uniqueness properties of solutions of initial and boundary value problems for differential equations as well as difference equations $[3,20]$. In further simplifying the proof of Opial's inequality, which has already been simplified by Olech [23], Beescak [5], Levison [19], Mallows [21], and Pederson [25], it is proved that if $x$ is real absolutely continuous on $(0, b)$ and with $x(0)=0$, then

$$
\int_{0}^{b}|x(t)|\left|x^{\prime}(t)\right| d t \leq \frac{b}{2} \int_{0}^{b}\left|x^{\prime}(t)\right|^{2} d t .
$$

(c) The Author(s) 2019. This article is distributed under the terms of the Creative Commons Attribution 4.0 International License (http://creativecommons.org/licenses/by/4.0/), which permits unrestricted use, distribution, and reproduction in any medium, provided you give appropriate credit to the original author(s) and the source, provide a link to the Creative Commons license, and indicate if changes were made. 
For a generalization of (1.1), Beesack [5] proved that if $x$ is an absolutely continuous function on $[a, X]$ with $x(a)=0$, then

$$
\int_{a}^{X}|x(t)|\left|x^{\prime}(t)\right| d t \leq \frac{1}{2} \int_{a}^{X} \frac{1}{r(t)} d t \int_{a}^{X} r(t)\left|x^{\prime}(t)\right|^{2} d t
$$

where $r(t)$ is a positive and continuous function with $\int_{a}^{X} \frac{d t}{r(t)}<\infty$, and if $x(b)=0$, then

$$
\int_{X}^{b}|x(t)|\left|x^{\prime}(t)\right| d t \leq \frac{1}{2} \int_{X}^{b} \frac{1}{r(t)} d t \int_{X}^{b} r(t)\left|x^{\prime}(t)\right|^{2} d t
$$

Yang [27] simplified Beesack's proof and extended inequality (1.3) and proved that: If $x$ is an absolutely continuous function on $(a, b)$ with $x(a)=0$, then

$$
\int_{a}^{b} q(t)|x(t)|\left|x^{\prime}(t)\right| d t \leq \frac{1}{2} \int_{a}^{b} \frac{1}{r(t)} d t \int_{a}^{b} r(t) q(t)\left|x^{\prime}(t)\right|^{2} d t
$$

where $r(t)$ is a positive and continuous function with $\int_{a}^{X} \frac{d t}{r(t)}<\infty$ and $q(t)$ is a positive bounded and nonincreasing function on $[a, b]$.

Hua [15] extended inequality (1.2) and proved that: If $x$ is an absolutely continuous function with $x(a)=0$, then

$$
\int_{a}^{b}|x(t)|^{p}\left|x^{\prime}(t)\right| d t \leq \frac{(b-a)^{p}}{p+1} \int_{a}^{b}\left|x^{\prime}(t)\right|^{p+1} d t
$$

where $p$ is a positive integer. We mentioned here that the result in [15] failed to apply for general values of $p$.

Maroni [22] generalized (1.3) and proved that: If $x$ is an absolutely continuous function on $[a, b]$ with $x(a)=0=x(b)$, then

$$
\int_{a}^{b}|x(t)|\left|x^{\prime}(t)\right| d t \leq \frac{1}{2}\left(\int_{a}^{b}\left(\frac{1}{r(t)}\right)^{\alpha-1} d t\right)^{\frac{2}{\alpha}}\left(\int_{a}^{b} r(t)\left|x^{\prime}(t)\right|^{v} d t\right)^{\frac{2}{v}}
$$

where $\int_{a}^{b}\left(\frac{1}{r(t)}\right)^{\alpha-1}<\infty, \alpha \geq 1$, and $\frac{1}{\alpha}+\frac{1}{v}=1$.

In fact, the discrete analogy of (1.1), which has been proved by Lasota [17], is given by

$$
\sum_{i=1}^{h-1}\left|x_{i} \Delta x_{i}\right| \leq \frac{1}{2}\left[\frac{h+1}{2}\right] \sum_{i=0}^{h-1}\left|\Delta x_{i}\right|^{2}
$$

where $\left\{x_{i}\right\}_{0 \leq i \leq h}$ is a sequence of real numbers with $x_{0}=x_{h}=0$. The discrete analogy of (1.2) is proved in [4, Theorem 5.2.2] and given by

$$
\sum_{i=1}^{h-1}\left|x_{i} \Delta x_{i}\right| \leq \frac{h-1}{2} \sum_{i=0}^{h-1}\left|\Delta x_{i}\right|^{2},
$$

where $\left\{x_{i}\right\}_{0 \leq i \leq h}$ is a sequence of real numbers with $x_{0}=0$.

In this paper, we are concerned with a certain class of Opial-type dynamic inequalities on time scales and their extensions. If the time scale equals the real (or the integers), the 
results represent the classical results for differential (or difference) inequalities. The three most popular examples of calculus on time scales are differential calculus, difference calculus, and quantum calculus (see Kac and Cheung [16]), that is, when $\mathbb{T}=\mathbb{R}, \mathbb{T}=\mathbb{N}$, and $\mathbb{T}=q^{\mathbb{N}}=\left\{q^{t}: t \in \mathbb{N}_{0}\right\}$, where $q>1$. For more details on time scale analysis, we refer the reader to the two books by Bohner and Peterson $[7,9]$ which summarize and organize much of the time scale calculus.

In [6], Bohner and Kaymakcalan introduced the dynamic Opial inequality on time scales, which unifies the continuous version (1.2) and the discrete version (1.9), and proved that if $x:[0, b] \cap \mathbb{T} \longrightarrow \mathbb{R}$ is delta differentiable with $x(0)=0$, then

$$
\int_{0}^{h}\left|x(t)+x^{\sigma}(t)\right|\left|x^{\Delta}(t)\right| \Delta t \leq h \int_{0}^{h}\left|x^{\Delta}(t)\right|^{2} \Delta t
$$

In the following, we recall some of the related results that have been established for differential inequalities and dynamic inequalities on time scales that serve and motivate the contents of this paper.

In 1966, Yang [27] proved the following inequality which is a generalization of Opial's inequality and some extensions of Beesack's. He proved that, if $u$ is an absolutely continuous function on $[a, X]$ with $u(a)=0$, then

$$
2 \int_{a}^{X} s(x)|u(x)|\left|u^{\prime}(x)\right| d x \leq \int_{a}^{X} \frac{d x}{l(x)} \int_{a}^{X} l(x) s(x) u^{\prime}(x)^{2} d x
$$

where $l$ is a positive and continuous function with $\int_{a}^{X} \frac{d x}{l(x)}<\infty$, and $s$ is bounded, positive, and nonincreasing on $[a, X]$.

Also, in the same paper, the author proved that, if $u(b)=0$, then

$$
2 \int_{X}^{b} s(x)|u(x)|\left|u^{\prime}(x)\right| d x \leq \int_{X}^{b} \frac{d x}{l(x)} \int_{X}^{b} l(x) s(x)\left[u^{\prime}(x)\right]^{2} d x,
$$

where $s$ is a bounded, positive, and nonincreasing function on $[X, b]$.

Also, as another extension of Opial's inequality, Yang [27] proved that: If $u(a)=u(b)=0$, then

$$
\int_{a}^{b}|u|^{p}\left|u^{\prime}\right|^{q} d x \leq \frac{q}{p+q}\left(\frac{b-a}{2}\right)^{p} \int_{a}^{b}\left|u^{\prime}\right|^{p+q} d x, \quad p, q \geq 1 .
$$

Cheng-Shyong Lee [18] generalized (1.11) and proved that: If $u$ is an absolutely continuous function on $[a, X]$ with $u(a)=0$, then

$$
\begin{aligned}
& (p+q) \int_{a}^{X} l^{q(q-1)}(x) s^{q}(x)|u(x)|^{p}\left|u^{\prime}(x)\right|^{q} d x \\
& \quad \leq q\left(\int_{a}^{X} l^{(-q)}(x) d x\right)^{p}\left(\int_{a}^{X} l^{q(p+q-1)}(x) s^{q}(x)\left|u^{\prime}(x)\right|^{(p+q)} d x\right)
\end{aligned}
$$

where $p \geq 0, q \geq 1$ and $l$ is positive with $\int_{a}^{X} \frac{d x}{l(x)}<\infty$, and $s$ is positive and nonincreasing on $[a, X]$. 
Also, in the same paper, the author gave a generalization of inequality (1.12) and proved that: If $u$ is an absolutely continuous function on $[X, b]$ with $u(b)=0$, then

$$
\begin{aligned}
& (p+q) \int_{X}^{b} l^{q(q-1)}(x) s^{q}(x)|u(x)|^{p}\left|u^{\prime}(x)\right|^{q} d x \\
& \quad \leq q\left(\int_{X}^{b} l^{(-q)}(x) d x\right)^{p}\left(\int_{X}^{b} l^{q(p+q-1)}(x) s^{q}(x)\left|u^{\prime}(x)\right|^{(p+q)} d x\right)
\end{aligned}
$$

where $p \geq 0, q \geq 1$, and $l$ is a positive and continuous function with $\int_{X}^{b} \frac{d x}{l(x)}<\infty$, and $s$ is positive and nondecreasing on $[X, b]$.

Also, Cheng-Shyong Lee in his paper extended inequality (1.13) and proved that: If $u$ is an absolutely continuous function on $[a, b]$ with $u(a)=u(b)=0$, then

$$
\begin{aligned}
& (p+q) \int_{a}^{b} l^{q(q-1)}(x) s^{q}(x)|u(x)|^{p}\left|u^{\prime}(x)\right|^{q} d x \\
& \quad \leq q\left(\int_{a}^{b} l^{(-q)}(x) d x\right)^{p}\left(\int_{a}^{b} l^{q(p+q-1)}(x) s^{q}(x)\left|u^{\prime}(x)\right|^{(p+q)} d x\right) .
\end{aligned}
$$

During the last decade, many inequalities have been studied by different authors (see [1, $2,10-14,26]$ and the references cited therein).

In this article, we will state and prove some dynamic Opial-type inequalities on time scales and the results obtained here generalize Theorems (1.14), (1.15), and (1.16). After each result, we will study as special cases when $\mathbb{T}=\mathbb{R}$ and $\mathbb{T}=\mathbb{N}$ to obtain some continuous and discrete results. The present article is arranged as follows: In Sect. 2, some basic concepts of the calculus on time scales and useful lemmas are introduced. In Sect. 3, we state and prove the main results. In Sect. 4, we write the conclusion of the paper.

\section{Preliminaries and letmmas on time scales}

Before proceeding to our main results, we mention some essentials on time scales and universal symbols to be used in this paper. From now on, $\mathbb{R}$ and $\mathbb{Z}$ denote the set of all real numbers and the set of all integers, respectively.

A time scale $\mathbb{T}$ is an arbitrary nonempty closed subset of $\mathbb{R}$. We suppose throughout the article that $\mathbb{T}$ has the topology that it inherits from the standard topology on $\mathbb{R}$. The forward jump operator $\sigma: \mathbb{T} \rightarrow \mathbb{T}$ is defined for any $t \in \mathbb{T}$ by

$$
\sigma(t):=\inf \{s \in \mathbb{T}: s>t\}
$$

and the backward jump operator $\rho: \mathbb{T} \rightarrow \mathbb{T}$ is defined for any $t \in \mathbb{T}$ by

$$
\rho(t):=\sup \{s \in \mathbb{T}: s<t\}
$$

In the previous two definitions, we set $\inf \emptyset=\sup \mathbb{T}$ (i.e., if $t$ is the maximum of $\mathbb{T}$, then $\sigma(t)=t$ ) and $\sup \emptyset=\inf \mathbb{T}$ (i.e., if $t$ is the minimum of $\mathbb{T}$, then $\rho(t)=t$ ), where $\emptyset$ is the empty set.

A point $t \in \mathbb{T}$ with inf $\mathbb{T}<t<\sup \mathbb{T}$ is said to be right-scattered if $\sigma(t)>t$, right-dense if $\sigma(t)=t$, left-scattered if $\rho(t)<t$, and left-dense if $\rho(t)=t$. Points that are simultaneously 
right-dense and left-dense are called dense points. Whereas points that are simultaneously right-scattered and left-scattered are called isolated points.

We define the forward graininess function $\mu: \mathbb{T} \rightarrow[0, \infty)$ for any $t \in \mathbb{T}$ by $\mu(t):=\sigma(t)-t$.

Let $f: \mathbb{T} \rightarrow \mathbb{R}$ be a function. Then the function $f^{\sigma}: \mathbb{T} \rightarrow \mathbb{R}$ is defined by $f^{\sigma}(t)=f(\sigma(t))$, $\forall t \in \mathbb{T}$, that is, $f^{\sigma}=f \circ \sigma$. In a similar manner, the function $f^{\rho}: \mathbb{T} \rightarrow \mathbb{R}$ is defined by $f^{\rho}(t)=f(\rho(t)), \forall t \in \mathbb{T}$, that is, $f^{\rho}=f \circ \rho$.

We introduce the set $\mathbb{T}^{\kappa}$ as follows: If $\mathbb{T}$ has a left-scattered maximum $t_{1}$, then $\mathbb{T}^{\kappa}=$ $\mathbb{T}-\left\{t_{1}\right\}$, otherwise $\mathbb{T}^{\kappa}=\mathbb{T}$.

The interval $[a, b]$ in $\mathbb{T}$ is defined by

$$
[a, b]_{\mathbb{T}}=\{t \in \mathbb{T}: a \leq t \leq b\}
$$

Open intervals and half-closed interval are defined similarly.

Suppose that $f: \mathbb{T} \rightarrow \mathbb{R}$ is a function and $t \in \mathbb{T}^{\kappa}$. Then we say that $f^{\Delta}(t) \in \mathbb{R}$ is the delta derivative of $f$ at $t$ if for any $\varepsilon>0$ there exists a neighborhood $U$ of $t$ such that, for all $s \in U$, we have

$$
\left|[f(\sigma(t))-f(s)]-f^{\Delta}(t)[\sigma(t)-s]\right| \leq \varepsilon|\sigma(t)-s|
$$

Furthermore, $f$ is said to be delta differentiable on $\mathbb{T}^{\kappa}$ if it is delta differentiable at each $t \in \mathbb{T}^{\kappa}$.

If $f, g: \mathbb{T} \rightarrow \mathbb{R}$ are delta differentiable functions at $t \in \mathbb{T}^{\kappa}$, then

(i) $(f+g)^{\Delta}(t)=f^{\Delta}(t)+g^{\Delta}(t)$;

(ii) $(f g)^{\Delta}(t)=f^{\Delta}(t) g(t)+f(\sigma(t)) g^{\Delta}(t)=f(t) g^{\Delta}(t)+f^{\Delta}(t) g(\sigma(t))$;

(iii) $\left(\frac{f}{g}\right)^{\Delta}(t)=\frac{f^{\Delta}(t) g(t)-f(t) g^{\Delta}(t)}{g(t) g(\sigma(t))}, g(t) g(\sigma(t)) \neq 0$.

A function $g: \mathbb{T} \rightarrow \mathbb{R}$ is called right-dense continuous (rd-continuous) if $g$ is continuous at the right-dense points in $\mathbb{T}$ and its left-sided limits exist at all left-dense points in $\mathbb{T}$.

A function $F: \mathbb{T} \rightarrow \mathbb{R}$ is said to be a delta antiderivative of $f: \mathbb{T} \rightarrow \mathbb{R}$ if $F^{\Delta}(t)=f(t)$ for all $t \in \mathbb{T}^{\kappa}$. In this case, the definite delta integral of $f$ is defined by

$$
\int_{a}^{b} f(t) \Delta t=F(b)-F(a) \quad \text { for all } a, b \in \mathbb{T} .
$$

If $g \in C_{r d}(\mathbb{T})$ and $t, t_{0} \in \mathbb{T}$, then the definite integral $G(t):=\int_{t_{0}}^{t} g(s) \Delta s$ exists, and $G^{\Delta}(t)=$ $g(t)$ holds.

Assume that $a, b, c \in \mathbb{T}, \alpha \in \mathbb{R}$, and $f, g$ are continuous functions on $[a, b]_{\mathbb{T}}$. Then

(i) $\int_{a}^{b}[f(t)+g(t)] \Delta t=\int_{a}^{b} f(t) \Delta t+\int_{a}^{b} g(t) \Delta t$;

(ii) $\int_{a}^{b} \alpha f(t) \Delta t=\alpha \int_{a}^{b} f(t) \Delta t$;

(iii) $\int_{a}^{b} f(t) \Delta t=\int_{a}^{c} f(t) \Delta t+\int_{c}^{b} f(t) \Delta t$;

(iv) $\int_{a}^{b} f(t) \Delta t=-\int_{b}^{a} f(t) \Delta t$;

(v) $\int_{a}^{a} f(t) \Delta t=0$;

(vi) if $f(t) \geq g(t)$ on $[a, b]_{\mathbb{T}}$, then $\int_{a}^{b} f(t) \Delta t \geq \int_{a}^{b} g(t) \Delta t$.

We will need the following important relations between calculus on time scales $\mathbb{T}$ and either continuous calculus on $\mathbb{R}$ or discrete calculus on $\mathbb{Z}$. Note that:

(i) If $\mathbb{T}=\mathbb{R}$, then

$$
\sigma(t)=t, \quad \mu(t)=0, \quad f^{\Delta}(t)=f^{\prime}(t), \quad \int_{a}^{b} f(t) \Delta t=\int_{a}^{b} f(t) d t .
$$


(ii) If $\mathbb{T}=\mathbb{Z}$, then

$$
\sigma(t)=t+1, \quad \mu(t)=1, \quad f^{\Delta}(t)=f(t+1)-f(t), \quad \int_{a}^{b} f(t) \Delta t=\sum_{t=a}^{b-1} f(t) .
$$

In the following, we present the basic theorems that will be needed in the proof of our main results.

Theorem 2.1 (Chain rule on time scales [8]) Assume $g: \mathbb{R} \rightarrow \mathbb{R}$ is continuous, $g: \mathbb{T} \rightarrow \mathbb{R}$ is delta differentiable on $\mathbb{T}^{\kappa}$, and $f: \mathbb{R} \rightarrow \mathbb{R}$ is continuously differentiable. Then there exists $c \in[t, \sigma(t)]$ with

$$
(f \circ g)^{\Delta}(t)=f^{\prime}(g(c)) g^{\Delta}(t)
$$

Theorem 2.2 (Chain rule on time scales [8]) Let $f: \mathbb{R} \rightarrow \mathbb{R}$ be continuously differentiable and suppose $g: \mathbb{T} \rightarrow \mathbb{R}$ is delta differentiable. Then $f \circ g: \mathbb{T} \rightarrow \mathbb{R}$ is delta differentiable and the formula

$$
(f \circ g)^{\Delta}(t)=\left\{\int_{0}^{1}\left[f^{\prime}\left(h g^{\sigma}(t)+(1-h) g(t)\right)\right] d h\right\} g^{\Delta}(t)
$$

holds.

Theorem 2.3 (Dynamic Hölder's inequality [7]) Let $a, b \in \mathbb{T}$ and $f, g \in C_{r d}\left([a, b]_{\mathbb{T}},[0, \infty)\right)$. If $p, q>1$ with $\frac{1}{p}+\frac{1}{q}=1$, then

$$
\int_{a}^{b} f(t) g(t) \Delta t \leq\left[\int_{a}^{b} f^{p}(t) \Delta t\right]^{\frac{1}{p}}\left[\int_{a}^{b} g^{q}(t) \Delta t\right]^{\frac{1}{q}}
$$

\section{Main results}

In this section, we state and prove our main results. Throughout this section, $p$ and $q$ are conjugate to each other, i.e., $1 / p+1 / q=1$.

Theorem 3.1 Let $\mathbb{T}$ be a time scale with $a, X \in \mathbb{T}$. Let $l$, s be positive $r d$-continuous functions with s nonincreasing and $\int_{a}^{X} l^{-q}(x) \Delta x<\infty$. Moreover, let $u$ be a rd-continuous function on $[a, X]_{\mathbb{T}}$ with $u(a)=0$. Then, for all $p \geq 1$ and $q \geq 1$, we have

$$
\begin{aligned}
& (p+q) \int_{a}^{X} l^{q(q-1)}(x) s^{q}(x)|u(x)|^{p}\left|u^{\Delta}(x)\right|^{q} \Delta x \\
& \quad \leq q\left(\int_{a}^{X} l^{-q}(x) \Delta x\right)^{p}\left(\int_{a}^{X} l^{q(p+q-1)}(x) s^{q}(x)\left|u^{\Delta}(x)\right|^{p+q} \Delta x\right) .
\end{aligned}
$$

Proof Define the function $z$ by the following:

$$
z(x)=\int_{a}^{x} l^{q(q-1)}(t) s^{q^{2} /(p+q)}(t)\left|u^{\Delta}(t)\right|^{q} \Delta t
$$

Using the fact that $z(a)=0$, we have

$$
z^{\Delta}(x)=l^{q(q-1)}(x) s^{q^{2} /(p+q)}(x)\left|u^{\Delta}(x)\right|^{q} .
$$


Since

$$
|u(x)|=|u(x)-u(a)|=\left|\int_{a}^{x} u^{\Delta}(t) \Delta t\right| \leq \int_{a}^{x}\left|u^{\Delta}(t)\right| \Delta t .
$$

By using the dynamic Hölder inequality (2.2) with indices $q$ and $q /(q-1)$, we deduce

$$
\begin{aligned}
|u(x)| & \leq \int_{a}^{x} l^{1-q}(t) l^{q-1}(t)\left|u^{\Delta}(t)\right| \Delta t \\
& \leq\left(\int_{a}^{x}\left(l^{1-q}(t)\right)^{q /(q-1)} \Delta t\right)^{(q-1) / q}\left(\int_{a}^{x}\left(l^{q-1}(t)\right)^{q}\left|u^{\Delta}(t)\right|^{q} \Delta t\right)^{1 / q} \\
& =\left(\int_{a}^{x} l^{-q}(t) \Delta t\right)^{(q-1) / q}\left(\int_{a}^{x} l^{q(q-1)}(t)\left|u^{\Delta}(t)\right|^{q} \Delta t\right)^{1 / q} .
\end{aligned}
$$

Therefore

$$
|u(x)|^{p} \leq\left(\int_{a}^{x} l^{-q}(t) \Delta t\right)^{p(q-1) / q}\left(\int_{a}^{x} l^{q(q-1)}(t)\left|u^{\Delta}(t)\right|^{q} \Delta t\right)^{p / q} .
$$

Since $s$ is positive and nonincreasing on $[a, X]_{\mathbb{T}}$, we have

$$
\begin{aligned}
s^{p q /(p+q)}(x)|u(x)|^{p} & \leq s^{p q /(p+q)}(x)\left(\int_{a}^{x} l^{-q}(t) \Delta t\right)^{p(q-1) / q}\left(\int_{a}^{x} l^{q(q-1)}(t)\left|u^{\Delta}(t)\right|^{q} \Delta t\right)^{p / q} \\
& \leq\left(\int_{a}^{X} l^{-q}(t) \Delta t\right)^{p(q-1) / q}\left(\int_{a}^{x} l^{q(q-1)}(t) s^{q^{2} /(p+q)}(t)\left|u^{\Delta}(t)\right|^{q} \Delta t\right)^{p / q} \\
& =\left(\int_{a}^{X} l^{-q}(t) \Delta t\right)^{p(q-1) / q} z^{p / q}(x) .
\end{aligned}
$$

Then multiplying the both sides of (3.2) by $l^{q(q-1)}(x) s^{q^{2} /(p+q)}(x)\left|u^{\Delta}(x)\right|^{q} \geq 0$, we get

$$
\begin{aligned}
& l^{q(q-1)}(x) s^{p q /(p+q)}(x) s^{q^{2} /(p+q)}(x)|u(x)|^{p}\left|u^{\Delta}(x)\right|^{q} \\
& \quad \leq l^{q(q-1)}(x)\left(\int_{a}^{X} l^{-q}(t) \Delta t\right)^{p(q-1) / q} z^{p / q}(x) s^{q^{2} /(p+q)}(x)\left|u^{\Delta}(x)\right|^{q} .
\end{aligned}
$$

Multiplying (3.3) by $(p+q)$ and integrating it from $a$ to $X$, we obtain

$$
\begin{aligned}
& (p+q) \int_{a}^{X} l^{q(q-1)}(x) s^{q}(x)|u(x)|^{p}\left|u^{\Delta}(x)\right|^{q} \Delta x \\
& \leq(p+q)\left(\int_{a}^{X} l^{-q}(x) \Delta t\right)^{p(q-1) / q} \int_{a}^{X} z^{p / q}(x) z^{\Delta}(x) \Delta x .
\end{aligned}
$$

Now, from the chain rule on time scales (2.1), we have

$$
\left(z^{(p+q) / q}\right)^{\Delta}(x)=\frac{p+q}{q} z^{p / q}(c) z^{\Delta}(x), \quad c \in[x, \sigma(x)] .
$$

Since $z^{\Delta}(x) \geq 0$ and $x \leq c$, we get

$$
\left(z^{(p+q) / q}\right)^{\Delta}(x)=\frac{p+q}{q} z^{p / q}(c) z^{\Delta}(x) \geq \frac{p+q}{q} z^{p / q}(x) z^{\Delta}(x),
$$


from (3.4) and (3.5), we have (note that $z(a)=0$ )

$$
\begin{aligned}
(p & +q) \int_{a}^{X} l^{q(q-1)}(x) s^{q}(x)|u(x)|^{p}\left|u^{\Delta}(x)\right|^{q} \Delta x \\
& \leq q\left(\int_{a}^{X} l^{-q}(x) \Delta x\right)^{p(q-1) / q}\left(\int_{a}^{X}\left[z^{(p+q) / q}(x)\right]^{\Delta} \Delta x\right) \\
& \leq q\left(\int_{a}^{X} l^{-q}(x) \Delta x\right)^{p(q-1) / q} z^{(p+q) / q}(X) \\
& =q\left(\int_{a}^{X} l^{-q}(x) \Delta x\right)^{p(q-1) / q}\left(\int_{a}^{X} l^{q(q-1)}(x) s^{q^{2} /(p+q)}(x)\left|u^{\Delta}(x)\right|^{q} \Delta x\right)^{(p+q) / q} .
\end{aligned}
$$

Applying the dynamic Hölder inequality (2.2) with indices $(p+q) / p$ and $(p+q) / q$, we obtain

$$
\begin{aligned}
& {\left[\int_{a}^{X} l^{q(q-1)}(x) s^{q^{2} /(p+q)}(x)\left|u^{\Delta}(x)\right|^{q} \Delta x\right]^{(p+q) / q} } \\
&=\left(\int_{a}^{X} l^{-p q /(p+q)}(x) l^{q^{2}\left(1-\frac{1}{p+q}\right)}(x) s^{q^{2}(p+q)}(x)\left|u^{\Delta}(x)\right|^{q} \Delta x\right)^{\frac{p+q}{q}} \\
& \leq {\left[\left(\int_{a}^{X}\left(l^{-p q /(p+q)}(x)\right)^{\frac{p+q}{p}} \Delta x\right)^{\frac{p}{p+q}}\right.} \\
&\left.\times\left(\int_{a}^{X}\left(l^{q^{2}\left(1-\frac{1}{p+q}\right)}(x) s^{q^{2} /(p+q)}(x)\left|u^{\Delta}(x)\right|^{q} \Delta x\right)^{\frac{p+q}{q}}\right)^{\frac{q}{p+q}}\right]^{\frac{p+q}{q}} \\
&=\left(\int_{a}^{X} l^{-q}(x) \Delta x\right)^{p / q}\left(\int_{a}^{X} l^{q(p+q-1)}(x) s^{q}(x)\left|u^{\Delta}(x)\right|^{p+q} \Delta x\right) .
\end{aligned}
$$

By combining (3.6) and (3.7), we obtain

$$
\begin{aligned}
& (p+q) \int_{a}^{X} l^{q(q-1)}(x) s^{q}(x)|u(x)|^{p}\left|u^{\Delta}(x)\right|^{q} \Delta x \\
& \quad \leq q\left(\int_{a}^{X} l^{-q}(x) \Delta x\right)^{p}\left(\int_{a}^{X} l^{q(p+q-1)}(x) s^{q}(x)\left|u^{\Delta}(x)\right|^{p+q} \Delta x\right)
\end{aligned}
$$

which is the required inequality (3.1). This completes the proof.

Corollary 3.2 When $\mathbb{T}=\mathbb{R}$, the inequality (3.1) reduces to the following inequality of Cheng-Shyong Lee [18, Theorem 1.1]

$$
\begin{aligned}
& (p+q) \int_{a}^{X} l^{q(q-1)}(x) s^{q}(x)|u(x)|^{p}\left|u^{\prime}(x)\right|^{q} d x \\
& \quad \leq q\left(\int_{a}^{X} l^{-q}(x) d x\right)^{p}\left(\int_{a}^{X} l^{q(p+q-1)}(x) s^{q}(x)\left|u^{\prime}(x)\right|^{p+q} d x\right) .
\end{aligned}
$$

Remark 3.3 If $\mathbb{T}=\mathbb{R}$ and take $p=q=1$, we get the following inequality of Yang [27, Theorem 3] which is a generalization of Opial's inequality

$$
2 \int_{a}^{X} s(x)|u(x)|\left|u^{\prime}(x)\right| d x \leq \int_{a}^{x} l^{-1}(x) d x \int_{a}^{X} l(x) s(x) u^{\prime 2}(x) d x .
$$


Corollary 3.4 If $\mathbb{T}=\mathbb{N}$. Assume that $p$, $q$ are positive real numbers such that $p \geq 0, q \geq 1$ and $\left\{l_{i}\right\}_{0 \leq i \leq N}$ is a nonnegative real sequence. If $\left\{u_{i}\right\}_{0 \leq i \leq N}$ is a sequence of real numbers with $u(0)=0$, then

$$
\begin{aligned}
(p & +q) \sum_{n=a}^{N-1} l^{q(q-1)}(n) s^{q}(n)|u(n)|^{p}|\Delta u(n)|^{q} \\
& \leq q\left(\sum_{n=a}^{N-1} l^{-q}(n)\right)^{p}\left(\sum_{n=a}^{N-1} l^{q(p+q-1)}(n) s^{q}(n)|\Delta u(n)|^{p+q}\right) .
\end{aligned}
$$

Theorem 3.5 Let $\mathbb{T}$ be a time scale with $X, b \in \mathbb{T}$. Let $l$, s be positive rd-continuous functions with s nondecreasing and $\int_{X}^{b} l^{-q}(x) \Delta x<\infty$. Moreover, let $u$ be an rd-continuous function on $[X, b]_{\mathbb{T}}$ with $u(b)=0$. Then, for all $p \geq 1$ and $q \geq 1$, we have

$$
\begin{aligned}
(p & +q) \int_{X}^{b} l^{q(q-1)}(x) s^{q}(x)\left|u^{\sigma}(x)\right|^{p}\left|u^{\Delta}(x)\right|^{q} \Delta x \\
& \leq q\left(\int_{X}^{b} l^{-q}(x) \Delta x\right)^{p}\left(\int_{X}^{b} l^{(q(p+q-1))}(x) s^{q}(x)\left|u^{\Delta}(x)\right|^{p+q} \Delta x\right) .
\end{aligned}
$$

Proof Define the function $z$ by the following:

$$
z(x)=-\int_{x}^{b} l^{q(q-1)}(t) s^{q^{2} /(p+q)}(t)\left|u^{\Delta}(t)\right|^{q} \Delta t
$$

Using the fact that $z(b)=0$, we have

$$
z^{\Delta}(x)=l^{q(q-1)}(x) s^{q^{2} /(p+q)}(x)\left|u^{\Delta}(x)\right|^{q} .
$$

Since $\left|u^{\sigma}(x)\right|=\left|-u^{\sigma}(x)\right|=\left|u(b)-u^{\sigma}(x)\right|=\left|\int_{\sigma(x)}^{b} u^{\Delta}(t) \Delta t\right| \leq \int_{\sigma(x)}^{b}\left|u^{\Delta}(t)\right| \Delta t$.

By using the dynamic Hölder inequality (2.2) with indices $q$ and $q /(q-1)$, we have

$$
\begin{aligned}
\left|u^{\sigma}(x)\right| & \leq \int_{\sigma(x)}^{b} l^{1-q}(t) l^{q-1}(t)\left|u^{\Delta}(t)\right| \Delta t \\
& \leq\left(\int_{\sigma(x)}^{b}\left(l^{1-q}(t)\right)^{q /(q-1)} \Delta t\right)^{(q-1) / q}\left(\int_{\sigma(x)}^{b}\left(l^{q-1}(t)\right)^{q}\left|u^{\Delta}(t)\right|^{q} \Delta t\right)^{1 / q} \\
& =\left(\int_{\sigma(x)}^{b} l^{-q}(t) \Delta t\right)^{(q-1) / q}\left(\int_{\sigma(x)}^{b} l^{q(q-1)}(t)\left|u^{\Delta}(t)\right|^{q} \Delta t\right)^{1 / q} .
\end{aligned}
$$

Therefore

$$
\left|u^{\sigma}(x)\right|^{p} \leq\left(\int_{\sigma(x)}^{b} l^{-q}(t) \Delta t\right)^{p(q-1) / q}\left(\int_{\sigma(x)}^{b} l^{q(q-1)}(t)\left|u^{\Delta}(t)\right|^{q} \Delta t\right)^{p / q}
$$

Since $s$ is positive and nondecreasing on $[X, b]_{\mathbb{T}}$, we have

$$
s^{p q /(p+q)}(x)\left|u^{\sigma}(x)\right|^{p} \leq s^{p q /(p+q)}(x)\left(\int_{\sigma(x)}^{b} l^{-q}(t) \Delta t\right)^{p(q-1) / q}\left(\int_{\sigma(x)}^{b} l^{q(q-1)}(t)\left|u^{\Delta}(t)\right|^{q} \Delta t\right)^{p / q}
$$




$$
\begin{aligned}
& \leq\left(\int_{X}^{b} l^{-q}(t) \Delta t\right)^{p(q-1) / q}\left(\int_{\sigma(x)}^{b} l^{q(q-1)}(t) s^{q^{2} /(p+q)}(t)\left|u^{\Delta}(t)\right|^{q} \Delta t\right)^{p / q} \\
& =\left(\int_{X}^{b} l^{-q}(t) \Delta t\right)^{p(q-1) / q}\left(-z^{\sigma}(x)\right)^{p / q} .
\end{aligned}
$$

Then multiplying the both sides of (3.9) by $l^{q(q-1)}(x) s^{q^{2} /(p+q)}(x)\left|u^{\Delta}(x)\right|^{q} \geq 0$, we get

$$
\begin{aligned}
& l^{q(q-1)}(x) s^{p q /(p+q)}(x) s^{q^{2} /(p+q)}(x)\left|u^{\sigma}(x)\right|^{p}\left|u^{\Delta}(x)\right|^{q} \\
& \quad \leq l^{q(q-1)}(x)\left(\int_{X}^{b} l^{-q}(t) \Delta t\right)^{p(q-1) / q}\left(-z^{\sigma}(x)\right)^{p / q} s^{q^{2} /(p+q)}(x)\left|u^{\Delta}(x)\right|^{q} .
\end{aligned}
$$

We can write (3.10) in the following form:

$$
l^{q(q-1)}(x) s^{q}(x)\left|u^{\sigma}(x)\right|^{p}\left|u^{\Delta}(x)\right|^{q} \Delta x \leq\left(\int_{X}^{b} l^{-q}(t) \Delta t\right)^{p(q-1) / q}\left(-z^{\sigma}(x)\right)^{p / q} z^{\Delta}(x) .
$$

Multiplying (3.11) by $(p+q)$ and integrating it from $X$ to $b$, we get

$$
\begin{aligned}
& (p+q) \int_{X}^{b} l^{q(q-1)}(x) s^{q}(x)\left|u^{\sigma}(x)\right|^{p}\left|u^{\Delta}(x)\right|^{q} \Delta x \\
& \leq(p+q)\left(\int_{X}^{b} l^{-q}(t) \Delta t\right)^{p(q-1) / q} \int_{X}^{b}\left(-z^{\sigma}(x)\right)^{p / q} z^{\Delta}(x) \Delta x .
\end{aligned}
$$

Now, from the chain rule on time scales (2.1), we have

$$
\left(-(-z)^{(p+q) / q}\right)^{\Delta}(x)=\frac{p+q}{q}(-z)^{p / q}(c) z^{\Delta}(x), \quad c \in[x, \sigma(x)] .
$$

Since $z^{\Delta}(x) \geq 0$ and $c \leq \sigma(x)$, we get

$$
\left(-(-z)^{(p+q) / q}\right)^{\Delta}(x)=\frac{p+q}{q}(-z)^{p / q}(c) z^{\Delta}(x) \geq \frac{p+q}{q}\left((-z)^{\sigma}(x)\right)^{p / q} z^{\Delta}(x) .
$$

From (3.12) and (3.13), we have (note that $z(b)=0$ )

$$
\begin{aligned}
(p & +q) \int_{X}^{b} l^{q(q-1)}(x) s^{q}(x)\left|u^{\sigma}(x)\right|^{p}\left|u^{\Delta}(x)\right|^{q} \Delta x \\
& \leq q\left(\int_{X}^{b} l^{-q}(x) \Delta x\right)^{p(q-1) / q}\left(\int_{X}^{b}\left[-(-z)^{(p+q) / q}(x)\right]^{\Delta} \Delta x\right) \\
& \leq q\left(\int_{X}^{b} l^{-q}(x) \Delta x\right)^{p(q-1) / q}(-z)^{(p+q) / q}(X) \\
& =q\left(\int_{X}^{b} l^{-q}(x) \Delta x\right)^{p(q-1) / q}\left(\int_{X}^{b} l^{q(q-1)}(x) s^{q^{2} /(p+q)}(x)\left|u^{\Delta}(x)\right|^{q} \Delta x\right)^{(p+q) / q} .
\end{aligned}
$$


Applying the dynamic Hölder inequality (2.2) with indices $(p+q) / p$ and $(p+q) / q$, we obtain

$$
\begin{aligned}
& {\left[\int_{X}^{b} l^{q(q-1)}(x) s^{q^{2} /(p+q)}(x)\left|u^{\Delta}(x)\right|^{q} \Delta x\right]^{(p+q) / q}} \\
& =\left(\int_{X}^{b} l^{-p q /(p+q)}(x) l^{q^{2}\left(1-\frac{1}{p+q}\right)}(x) s^{q^{2}(p+q)}(x)\left|u^{\Delta}(x)\right|^{q} \Delta x\right)^{\frac{p+q}{q}} \\
& \leq \quad\left[\left(\int_{X}^{b}\left(l^{-p q /(p+q)}(x) \Delta x\right)^{\frac{p+q}{p}}\right)^{\frac{p}{p+q}}\right. \\
& \left.\quad \times\left(\int_{X}^{b}\left(l^{q^{2}\left(1-\frac{1}{p+q}\right)}(x) s^{q^{2} /(p+q)}(x)\left|u^{\Delta}(x)\right|^{q} \Delta x\right)^{\frac{p+q}{q}}\right)^{\frac{q}{p+q}}\right]^{\frac{p+q}{q}} \\
& =\left(\int_{X}^{b} l^{-q}(x) \Delta x\right)^{p / q}\left(\int_{X}^{b} l^{q(p+q-1)}(x) s^{q}(x)\left|u^{\Delta}(x)\right|^{p+q} \Delta x\right) .
\end{aligned}
$$

By combining (3.14) and (3.15), we obtain

$$
\begin{aligned}
& (p+q) \int_{X}^{b} l^{q(q-1)}(x) s^{q}(x)\left|u^{\sigma}(x)\right|^{p}\left|u^{\Delta}(x)\right|^{q} \Delta x \\
& \quad \leq q\left(\int_{X}^{b} l^{-q}(x) \Delta x\right)\left(\int_{X}^{b} l^{q(p+q-1)}(x) s^{q}(x)\left|u^{\Delta}(x)\right|^{p+q} \Delta x\right),
\end{aligned}
$$

which is the desired inequality (3.8). This completes the proof.

Corollary 3.6 When $\mathbb{T}=\mathbb{R}$, inequality (3.8) reduces to the following inequality of ChengShyong Lee [18, Theorem 1.2]

$$
\begin{aligned}
& (p+q) \int_{X}^{b} l^{q(q-1)}(x) s^{q}(x)|u(x)|^{p}\left|u^{\prime}(x)\right|^{q} d x \\
& \quad \leq q\left(\int_{X}^{b} l^{-q}(x) d x\right)^{p}\left(\int_{X}^{b} l^{q(p+q-1)}(x) s^{q}(x)\left|u^{\prime}(x)\right|^{p+q} d x\right) .
\end{aligned}
$$

Remark 3.7 If $\mathbb{T}=\mathbb{R}$ and take $p=q=1$, we get the following inequality of Yang [27, Theorem 3] which is a generalization of Opial's inequality

$$
2 \int_{x}^{b} s(x)|u(x)|\left|u^{\prime}(x)\right| d x \leq \int_{x}^{b} l^{-1}(x) d x \int_{x}^{b} l(x) s(x) u^{\prime 2}(x) d x
$$

Corollary 3.8 When $\mathbb{T}=\mathbb{N}$. Assume that $p$, $q$ are positive real numbers such that $p \geq 0$, $q \geq 1$ and $\left\{l_{i}\right\}_{0 \leq i \leq N}$ is a nonnegative real sequence. If $\left\{u_{i}\right\}_{0 \leq i \leq N}$ is a sequence of real numbers with $u(0)=0$, then

$$
\begin{aligned}
(p & +q) \sum_{n=N}^{b-1} l^{q(q-1)}(n) s^{q}(n)|u(n+1)|^{p}|\Delta u(n)|^{q} \\
& \leq q\left(\sum_{n=N}^{b-1} l^{-q}(n)\right)^{p}\left(\sum_{n=N}^{b-1} l^{q(p+q-1)}(n) s^{q}(n)|\Delta u(n)|^{p+q}\right) .
\end{aligned}
$$


Theorem 3.9 Let $\mathbb{T}$ be a time scale with $a, b \in \mathbb{T}$. Let $l$, s be positive rd-continuous functions with $s$ nonincreasing on $[a, X]_{\mathbb{T}}$ and nondecreasing on $[X, b]_{\mathbb{T}}$. Let $K=\left(\int_{a}^{X} l^{-q}(x) \Delta x\right)^{p}=$ $\left(\int_{X}^{b} l^{-q}(x) \Delta x\right)^{p}$. Moreover, let $u$ be a nondecreasing rd-continuous function on $[a, b]_{\mathbb{T}}$ with $u(a)=u(b)=0$. Then, for all $p \geq 1$ and $q \geq 1$, we have

$$
\begin{gathered}
(p+q) \int_{a}^{b} l^{q(q-1)}(x) s^{q}(x)|u(x)|^{p}\left|u^{\Delta}(x)\right|^{q} \Delta x \\
\leq q K \int_{a}^{b} l^{q(p+q-1)}(x) s^{q}(x)\left|u^{\Delta}(x)\right|^{p+q} \Delta x .
\end{gathered}
$$

Proof

$$
\begin{aligned}
(p+q) & \int_{a}^{b} l^{q(q-1)}(x) s^{q}(x)|u(x)|^{p}\left|u^{\Delta}(x)\right|^{q} \Delta x \\
= & (p+q) \int_{a}^{X} l^{q(q-1)}(x) s^{q}(x)|u(x)|^{p}\left|u^{\Delta}(x)\right|^{q} \Delta x \\
& +(p+q) \int_{X}^{b} l^{q(q-1)}(x) s^{q}(x)|u(x)|^{p}\left|u^{\Delta}(x)\right|^{q} \Delta x .
\end{aligned}
$$

Since $u$ is nondecreasing and $\sigma(x) \geq x$, then $u^{\sigma}(x) \geq u(x)$. From (3.1), (3.8), and (3.17), we get

$$
\begin{aligned}
(p+q) & \int_{a}^{b} l^{q(q-1)}(x) s^{q}(x)|u(x)|^{p}\left|u^{\Delta}(x)\right|^{q} \Delta x \\
\leq & (p+q) \int_{a}^{X} l^{q(q-1)}(x) s^{q}(x)|u(x)|^{p}\left|u^{\Delta}(x)\right|^{q} \Delta x \\
& +(p+q) \int_{X}^{b} l^{q(q-1)}(x) s^{q}(x)\left|u^{\sigma}(x)\right|^{p}\left|u^{\Delta}(x)\right|^{q} \Delta x \\
\leq & q\left(\int_{a}^{X} l^{-q}(x) \Delta x\right)^{p}\left(\int_{a}^{X} l^{q(p+q-1)}(x) s^{q}(x)\left|u^{\Delta}(x)\right|^{p+q} \Delta x\right) \\
& +q\left(\int_{X}^{b} l^{-q}(x) \Delta x\right)\left(\int_{X}^{b} l^{q(p+q-1)}(x) s^{q}(x)\left|u^{\Delta}(x)\right|^{p+q} \Delta x\right) \\
= & q K\left(\int_{a}^{X} l^{q(p+q-1)}(x) s^{q}(x)\left|u^{\Delta}(x)\right|^{p+q} \Delta x+\int_{X}^{b} l^{q(p+q-1)}(x) s^{q}(x)\left|u^{\Delta}(x)\right|^{p+q} \Delta x\right) \\
= & q K \int_{a}^{b} l^{q(p+q-1)}(x) s^{q}(x)\left|u^{\Delta}(x)\right|^{p+q} \Delta x,
\end{aligned}
$$

which is the desired inequality (3.16). This completes the proof.

Corollary 3.10 When $\mathbb{T}=\mathbb{R}$, we get the following inequality of Cheng-Shyong Lee $[18$, Theorem 2]

$$
(p+q) \int_{a}^{b} l^{q(q-1)}(x) s^{q}(x)|u(x)|^{p}\left|u^{\prime}(x)\right|^{q} d x \leq q K \int_{a}^{b} l^{q(p+q-1)}(x) s^{q}(x)\left|u^{\prime}(x)\right|^{p+q} d x .
$$

Corollary 3.11 When $\mathbb{T}=\mathbb{N}$. Assume that $p$, $q$ are positive real numbers such that $p \geq 0$, $q \geq 1$ and $\left\{l_{i}\right\}_{0 \leq i \leq N}$ is a nonnegative real sequence. If $\left\{u_{i}\right\}_{0 \leq i \leq N}$ is a sequence of real numbers 
with $u(0)=0$, then

$$
(p+q) \sum_{n=a}^{b-1} l^{q(q-1)}(n) s^{q}(n)|u(n)|^{p}|\Delta u(n)|^{q} \leq q K\left(\sum_{n=a}^{b-1} l^{q(p+q-1)}(n) s^{q}(n)|\Delta u(n)|^{p+q}\right) .
$$

\title{
4 Conclusion
}

We have proved some new generalizations of dynamic Opial-type inequalities on time scales. As special cases of these dynamic inequalities, we obtained some integral and discrete inequalities known in the literature and also extended some existing dynamic inequalities on time scales.

\author{
Acknowledgements \\ The authors wish to express their sincere appreciation to the editor and the anonymous referees for their valuable \\ comments and suggestions.
}

\section{Funding}

Not applicable.

\section{Competing interests}

The authors declare that they have no financial and non-financial competing interests.

\section{Authors' contributions}

All authors contributed equally. All the authors read and approved the final manuscript.

\section{Author details}

'Department of Mathematics, Faculty of Women for (Art, Science, and Education), Ain Shams University, Cairo, Egypt. ${ }^{2}$ Department of Mathematics, Faculty of Science, Al-Azhar University, Cairo, Egypt. ${ }^{3}$ Community College, Shaqra University, Al-Dawadmi, Saudi Arabia. ${ }^{4}$ Department of Mathematics and Computer Science, Faculty of Science, Port Said University, Port Said, Egypt. ${ }^{5}$ Department of Mathematics, Princess Nourah bint Abdulrahman University, Riyadh, Kingdom of Saudi Arabia.

\section{Publisher's Note}

Springer Nature remains neutral with regard to jurisdictional claims in published maps and institutional affiliations.

Received: 28 January 2019 Accepted: 28 July 2019 Published online: 12 August 2019

\section{References}

1. Abdeldaim, A., El-Deeb, A.A.: On generalized of certain retarded nonlinear integral inequalities and its applications in retarded integro-differential equations. Appl. Math. Comput. 256, 375-380 (2015) MR 3316076

2. Abdeldaim, A., El-Deeb, A.A., Agarwal, P., El-Sennary, H.A.: On some dynamic inequalities of Steffensen type on time scales. Math. Methods Appl. Sci. 41(12), 4737-4753 (2018) MR 3828354

3. Agarwal, R.P., Lakshmikantham, V.: Uniqueness and Nonuniqueness Criteria for Ordinary Differential Equations. Series in Real Analysis, vol. 6. World Scientific, Singapore (1993)

4. Agarwal, R.P., Pang, P.Y.H.: Opial Inequalities with Applications in Differential and Difference Equations, vol. 320. Kluwer Academic, Dordrecht (1995) MR 1340422

5. Beesack, P.R.: On an integral inequality of Z. Opial. Transl. Am. Math. Soc. 104, 470-475 (1962)

6. Bohner, M., Kaymakcalan, B.: Opial inequalities on time scales. Ann. Pol. Math. 77(1), 11-20 (2001)

7. Bohner, M., Peterson, A.: Dynamic Equations on Time Scales: An Introduction with Applications. Birkhauser, Boston (2001)

8. Bohner, M., Peterson, A.: An Introduction with Applications. Dynamic Equations on Time Scales. Birkhäuser Boston, Boston (2001) MR 1843232

9. Bohner, M., Peterson, A.: Advances in Dynamic Equations on Time Scales. Birkhauser, Boston (2003)

10. El-Deeb, A.A.: Some Gronwall-Bellman type inequalities on time scales for Volterra-Fredholm dynamic integral equations. J. Egypt. Math. Soc. 26(1), Article 1 (2018)

11. El-Deeb, A.A., Cheung, W.-S.: A variety of dynamic inequalities on time scales with retardation. J. Nonlinear Sci. Appl. 11(10), 1185-1206 (2018) MR 3845535

12. El-Deeb, A.A., Elsennary, H.A., Cheung, W.-S.: Some reverse Hölder inequalities with Specht's ratio on time scales. J. Nonlinear Sci. Appl. 11(4), 444-455 (2018) MR 3780318

13. El-Deeb, A.A., Elsennary, H.A., Nwaeze, E.R.: Generalized weighted Ostrowski, trapezoid and Grüss type inequalities on time scales. Fasc. Math. 60, 123-144 (2018) MR 3846782

14. El-Deeb, A.A., Xu, H., Abdeldaim, A., Wang, G.: Some dynamic inequalities on time scales and their applications. Adv. Differ. Equ. 2019, 130 (2019) MR 3934717

15. Hua, L.K.: On an inequality of Opial. Sci. Sin. 14, 789-790 (1965)

16. Kac, V., Cheung, P.: Quantum Calculus, 1st edn. Springer, New York (2001) 
17. Lasota, A.A.: A discrete boundary value problem. Ann. Pol. Math. 20, 183-190 (1968)

18. Lee, C.-S.: On some generalization of inequalities of Opial, Yang and Shum. Can. Math. Bull. 23(1), 71-80 (1980)

19. Levinson, N.: On an inequality of Opial and Beesack. Proc. Am. Math. Soc. 15, 565-666 (1964)

20. Li, J.D.: Opial-type integral inequalities involving several higher order derivatives. J. Math. Anal. Appl. 167(1), 98-110 (1992)

21. Mallows, C.L.: An even simpler proof of Opial inequality. Proc. Am. Math. Soc. 16, 173 (1965)

22. Maroni, P.: Sur I' inegalite d'Opial-Beesack. C. R. Acad. Sci. 264, A62-A64 (1967)

23. Olech, C.: A simple proof of a certain result of Z. Opial. Ann. Pol. Math. 8, 61-63 (1960)

24. Opial, Z:: Sur une inégalité. Ann. Pol. Math. 8(1), 29-32 (1960)

25. Pederson, R.N.: On an inequality of Opial, Beesack and Levinson. Proc. Am. Math. Soc. 16, 174 (1965)

26. Tian, Y., El-Deeb, A.A., Meng, F.: Some nonlinear delay Volterra-Fredholm type dynamic integral inequalities on time scales. Discrete Dyn. Nat. Soc. 2018, Article ID 5841985 (2018). MR 3847518

27. Yang, G.S.: On a certain result of Z. Opial. Proc. Jpn. Acad. 42, 78-83 (1966)

\section{Submit your manuscript to a SpringerOpen ${ }^{\circ}$} journal and benefit from:

- Convenient online submission

- Rigorous peer review

- Open access: articles freely available online

- High visibility within the field

- Retaining the copyright to your article

Submit your next manuscript at $\gg$ springeropen.com 